\title{
Ultraviolet $A$ and Ultraviolet C Light-Induced Reduction of Surface Hydrocarbons on Titanium Implants
}

\author{
Zaheer Naauman ${ }^{1,2,3}$ \\ Zainul Ahmad Bin Rajion ${ }^{4}$ \\ H. A. Razak Noor ${ }^{7}$ \\ ${ }^{1}$ School of Dental Sciences, Health Campus, Universiti Sains \\ Malaysia, Kelantan, Malaysia \\ 2Department of Oral Biology, Institute of Dentistry, CMH Lahore \\ Medical College, Lahore, Pakistan \\ ${ }^{3}$ National University of Medical Sciences, Lahore, Pakistan \\ ${ }^{4}$ Department of Oral Maxillofacial Imaging, School of Dental Sciences, \\ Health Campus, Universiti Sains Malaysia, Kelantan, Malaysia \\ ${ }^{5}$ Department of Oral Biology, Rashid Latif Dental College, Lahore, \\ Pakistan \\ 6Universiti Sains Malaysia's Science Officer Society, Science and \\ Engineering Research Centre, Engineering Campus, Universiti Sains \\ Malaysia, Penang, Malaysia \\ ${ }^{7}$ Department in Oral and Maxillofacial Surgery, School of Dental \\ Sciences, Health Campus, Universiti Sains Malaysia, Kelantan, Malaysia
}

Shahbaz Maliha ${ }^{5}$ Pauzi Hariy ${ }^{6}$

Q. Saeed Muhammad 2,3

\begin{abstract}
Address for correspondence Zainul Ahmad Bin Rajion, BDS, Grad Dip Clin Dent, PhD. Department of Oral Maxillofacial Imaging, School of Dental Sciences, Health Campus, Universiti Sains Malaysia, Kelantan, Malaysia (e-mail: zainulrajion@usm.my).
\end{abstract}

Eur J Dent 2019;13:114-118

Abstract
Keywords
- photofunctionalization
- sandblasted acid
etched
- ultraviolet A
irradiation
- ultraviolet C
irradiation
- X-ray photoelectron
spectroscopy

Objective The carbon, titanium, and oxygen levels on titanium implant surfaces with or without ultraviolet (UV) pretreatment were evaluated at different wavelengths through X-ray photoelectron spectroscopy (XPS).

Materials and Methods This interventional experimental study was conducted on nine Dio UFII implants with hybrid sandblasted and acid-etched (SLA) surface treatments, divided equally into three groups. Control group A samples were not given UV irradiation, while groups B and C samples were given UVA (382 nm, $25 \mathrm{mWcm}^{2}$ ) and UVC (260 nm, $\left.15 \mathrm{mWcm}^{2}\right)$ irradiation, respectively. The atomic ratio of carbon, titanium, and oxygen was compared through XPS.

Results Mean carbon-to-titanium ratio and C1 peaks considerably increased in Group A compared to those in experimental Groups B and C. The intensity of Ti2p and O1s peaks was more pronounced for group C compared to that for groups $A$ and $B$.

Conclusions Although the decrease in surface hydrocarbons was the same in both UV-treated groups, the peak intensity of oxygen increased in the UVC-treated group. Thus, it can be concluded that compared with UVA irradiation, UVC irradiation has the potential to induce more hydrophilicity on SLA-coated implants.

\section{Introduction}

Commercially available implants are inevitably contaminated with hydrocarbons in their packaging, ${ }^{1}$ with an average percentage ranging from $17.9 \%$ to $76.5 \% .^{2}$ Increase in carbon content decreases the hydrophilicity, thus increasing the chances of implant failure. ${ }^{3,4}$ Pretreatment of dental implants either with ultraviolet (UV) irradiation (photofunctionalization) or with nonthermal plasma treatment $^{5}$ effectively removes the carboxylic groups, thus resulting in superhydrophilic surface. ${ }^{6,7}$ However, UV radiation showed superiority, particularly on titanium implants. ${ }^{6,8}$ Thus, in the present study, higher and lower wavelengths, UVA and UVC radiations, respectively, were employed on titanium implants as it is difficult to evaluate the optimum wavelength for photofunctionalization. 


\section{Materials and Methods}

An interventional experimental study was conducted at the Science and Engineering Research Centre Engineering Campus, Universiti Sains Malaysia (USM), on hybrid SLAcoated Dio UFII dental implants. A total of nine implants, each $10 \mathrm{~mm}$ long and $4.0 \mathrm{~mm}$ in diameter, were acquired for X-ray photoelectron spectroscopy (XPS) analysis. The Human Research Ethics Committee USM approved the study protocol. A purposive (nonprobability) sampling technique was adopted. Three replicate samples were required to analyze the objectives.

\section{Ultraviolet Irradiation}

Nine SLA-coated Dio UFII hybrid implants of identical dimensions were randomly divided into three groups with three samples in each group. Group A was the control group, whereas groups B and C samples were photofunctionalized for $10 \mathrm{~min}$ with UVA ( $382 \mathrm{~nm}, 25 \mathrm{~mW} / \mathrm{cm}^{2}$ ) and UVC ( $260 \mathrm{~nm}$, $\left.15 \mathrm{~mW} / \mathrm{cm}^{2}\right)$, respectively, by placing them in a UVACUBE 100 (Honle, Germany). Since UVA has the potential to penetrate through glass and clouds to change chemical bonds, while UVC does not, ${ }^{9}$ implants were removed from glass or plastic containers before UV irradiation to ensure exposure to all wavelengths.

\section{X-Ray Photoelectron Spectroscopy Analysis}

After irradiation, the titanium implant surface was evaluated using XPS. The atomic carbon/titanium (C/Ti) ratio of titanium implants was compared among the study groups. Representative XPS profiles of carbon, titanium, and oxygen contents on titanium implant surfaces were analyzed. ${ }^{10}$

The XPS wide and narrow scan spectra were acquired using AXIS Ultra Delay-Line Detector (DLD), Kratos, equipped with an $\mathrm{Al} \mathrm{K \alpha}$ X-ray source (1486.6 eV) at $10 \mathrm{~mA}$ and $15 \mathrm{kV}$, analyzing a $300 \mu \mathrm{m} \times 700 \mu \mathrm{m}$ area under a 4.8 EX10-9 Torr Ultravacuum environment inside a sample analysis chamber. Using the multichannel plate and DLD with a takeoff angle of $90^{\circ}$ and an acceptance angle of $30^{\circ}$, the analyses were performed in the hybrid lens mode with the slot aperture, and the analyzer was operated in fixed analyzer transmission mode with the pass energy of the hemispherical analyzer set at $160 \mathrm{eV}$ for the survey/wide scan and $20 \mathrm{eV}$ for the high-resolution scans/narrow scans. All the scans were required under charge neutralization conditions using a low-energy electron gun within the field magnetic lens.

The spectra were analyzed using Vision software (AXIS Ultra DLD, Kratos), which included vision manager and vision processing. The linear method was used for background subtraction and curve fitting. Binding energy was referenced to an adventitious carbon at $284.6 \mathrm{eV}$.

\section{Statistical Analysis}

SPSS version 20.0 (SPSS, Inc, Chicago, Illinois) was used for the data entry and analysis. The mean difference in the concentration of carbon, titanium, nitrogen, and oxygen, as well as the difference in C/Ti ratio among the three groups, was determined through one-way analysis of variance. The post hoc Tukey's test was used for multiple comparisons. $P<0.05$ was considered statistically significant and $P>0.05$ statistically nonsignificant.

\section{Results}

The average percentage of carbon for group A was highest at approximately $51.11 \pm 12.4$, compared to groups B $(25.8 \pm 0.27)$ and $C(26.37 \pm 0.84 ; P<0.01)$ (-Table 1). When compared group-wise, the difference between groups $B$ and C was insignificant (-Table 2 and $\boldsymbol{- F i g . ~ 1 ) . ~}$

Average titanium and oxygen percentages were significantly lower in group A and higher in groups B and $C(P<$ 0.01 ) ( - Table 1); there was no significant difference between Groups B and C (-Tables 1, 2 and - Fig. 1). Nitrogen percentages were similar among all groups (-Table $\mathbf{1}$ ).

Mean C/Ti ratios ( - Table $\mathbf{1}$ ) were significantly higher in group A than in UV-treated groups $(P<0.05)$, with no significant difference among groups $B$ and $C$ ( - Table 2 and $\boldsymbol{\sim}$ Fig. $\mathbf{1}$ ).

XPS spectra showed Ti2p, 01s, and C1s peaks for titanium surfaces in each group (-Figs. 2-4). In C1s XPS spectra ( - Fig. 3), the predominant peak attributed to hydrocarbon (C-C, C-H) at $284.7 \mathrm{eV}$ and the other three peaks (at 286.531, 288.241, and $288.954 \mathrm{eV}$, representing $\mathrm{C}-\mathrm{O}, \mathrm{C}=\mathrm{O}$, and $\mathrm{O}-\mathrm{C}=\mathrm{O}$, respectively) were significantly reduced after UVA and UVC treatment (-Fig. 3).

Table 1 Average levels of concentration for various components and ratio and comparison among the three groups (one-way analysis of variance)

\begin{tabular}{|l|l|l|l|l|}
\hline \multirow{2}{*}{} & \multicolumn{3}{|c|}{ Group, mean \pm SD } & \multirow{2}{*}{ P (ANOVA) } \\
\cline { 2 - 4 } & Control & UVA & UVC & \\
\hline Carbon & $51.11(12.40)$ & $25.80(0.27)$ & $26.37(0.84)$ & $0.008^{\dagger}$ \\
\hline Titanium & $14.35(4.06)$ & $22.29(0.50)$ & $21.81(0.55)$ & $0.011^{*}$ \\
\hline Nitrogen & $1.15(0.23)$ & $0.82(0.26)$ & $0.81(0.40)$ & 0.353 \\
\hline Oxygen & $33.39(8.70)$ & $51.33(0.11)$ & $51.02(0.68)$ & $0.007^{\dagger}$ \\
\hline Carbon/titanium ratio & $3.90(1.81)$ & $1.16(0.03)$ & $1.21(0.07)$ & $0.029^{*}$ \\
\hline
\end{tabular}

Abbreviations: ANOVA, analysis of variance; SD, standard deviation; UVA, ultraviolet A; UVC, ultraviolet C.

* The mean difference is significant at the 0.05 level.

tThe mean difference is significant at the 0.01 level.

Data are expressed as mean and SD $(n=3)$. 
Table 2 Group-wise comparison for components using Tukey's test

\begin{tabular}{|c|c|c|c|c|c|}
\hline Dependent variable & Group (I) & Group (J) & Mean difference (I-J) & SE & $p$ \\
\hline \multirow[t]{3}{*}{ Carbon } & \multirow[t]{2}{*}{ Control } & UVA & $25.31^{*}$ & 5.86 & 0.012 \\
\hline & & UVC & $24.74^{*}$ & 5.86 & 0.013 \\
\hline & UVA & UVC & -0.57 & 5.86 & 0.995 \\
\hline \multirow[t]{3}{*}{ Titanium } & \multirow[t]{2}{*}{ Control } & UVA & $-7.94^{*}$ & 1.95 & 0.015 \\
\hline & & UVC & $-7.46^{*}$ & 1.95 & 0.020 \\
\hline & UVA & UVC & 0.48 & 1.95 & 0.967 \\
\hline \multirow[t]{3}{*}{ Oxygen } & \multirow[t]{2}{*}{ Control } & UVA & $-17.94^{*}$ & 4.11 & 0.011 \\
\hline & & UVC & $-17.63^{*}$ & 4.11 & 0.012 \\
\hline & UVA & UVC & 0.31 & 4.11 & 0.997 \\
\hline \multirow[t]{3}{*}{ Carbon/titanium ratio } & \multirow[t]{2}{*}{ Control } & UVA & $2.75^{*}$ & 0.85 & 0.042 \\
\hline & & UVC & $2.69^{*}$ & 0.85 & 0.045 \\
\hline & UVA & UVC & -0.05 & 0.85 & 0.998 \\
\hline
\end{tabular}

Abbreviations: SD: standard deviation, SE: standard error, UVA: ultraviolet A, UVC: ultraviolet C.

*The mean difference is significant at the 0.05 level.

The data are expressed as mean and SD $(n=3)$.

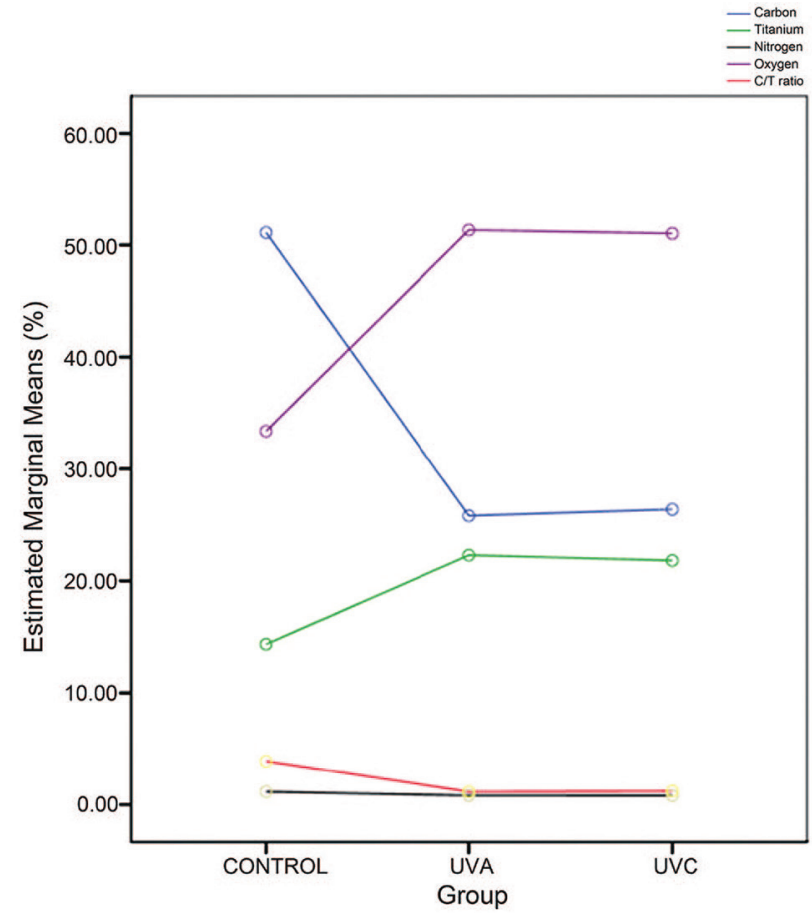

Fig. 1 Line graph presents average percentage for each component and carbon/titanium ratio for the three groups. The data are expressed as mean \pm standard deviation $(n=3)$.

For Ti2p spectra ( - Fig. 2 ), the titanium peaks were slightly more intense for group $\mathrm{C}$ at $458.5 \mathrm{eV}$ compared with those for groups A and B.

For 01s spectra (-Fig. 4), the first peak (predominant peak at $529.94 \mathrm{eV}$ ) was assigned to $01 \mathrm{~s}$ in $\mathrm{TiO}_{2}(\mathrm{O}-2)$, whereas the second peak was assigned for $01 \mathrm{~s}$ in $\mathrm{C}-\mathrm{O}$ and $\mathrm{C}=\mathrm{O}$ at $5531.262 \mathrm{eV}$, while the third peak at $531.837 \mathrm{eV}$ indicated the $01 \mathrm{~s}$ in titanium hydroxide $(\mathrm{Ti}-\mathrm{OH})$ and $\mathrm{O}-\mathrm{C}=\mathrm{O}$. All the three 01s peaks in XPS spectra showed an increasing trend of 01s after UVA and UVC treatment. The significant increase

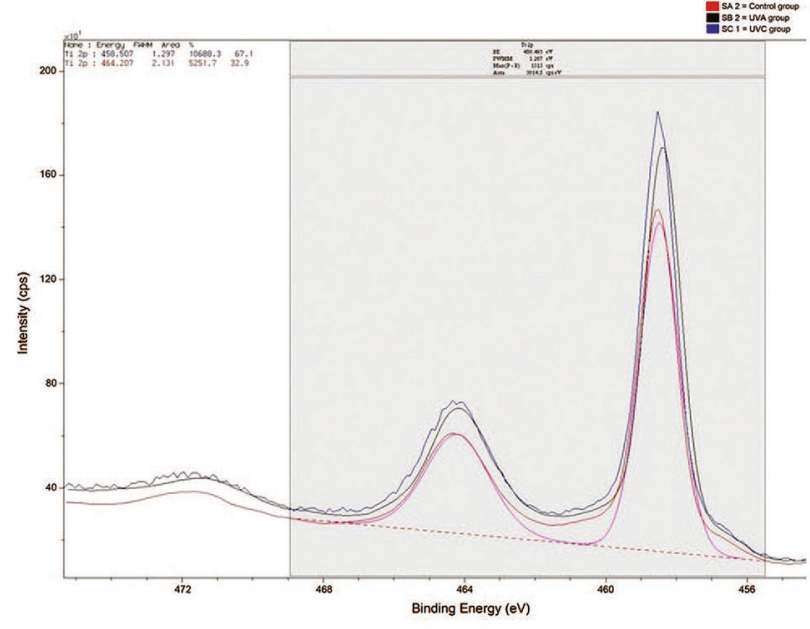

Fig. 2 X-ray photoelectron spectroscopy high-resolution spectra of the titanium surfaces of all the three groups showing Ti2p profile.

in peak intensity at $531.837 \mathrm{eV}$ was more pronounced for Group C than for Groups A and B.

\section{Discussion}

This study proved that high carbon levels are generally detected on commercial implant surfaces, indicating that exposing implants to UV radiation remove the surface hydrocarbons, thereby exposing the $\mathrm{OH}$ and oxygen radicals, resulting in a superhydrophilic surface, which attracts water molecules, proteins, and bone-forming cells. ${ }^{8}$ Therefore, hydrophilicity is indeed dependent on the carbon and oxygen ratios, with greater the carbon content, lesser the hydrophilicity, and vice versa. Previously, it has been reported that UVA and UVC irradiations of acid-etched implants for approximately $48 \mathrm{~h}$ at 0.1 and $2 \mathrm{~mW} / \mathrm{cm}^{2}$, respectively, continuously decreased the atomic percentage of carbon from $50 \%$ to $<20 \%$ in both 


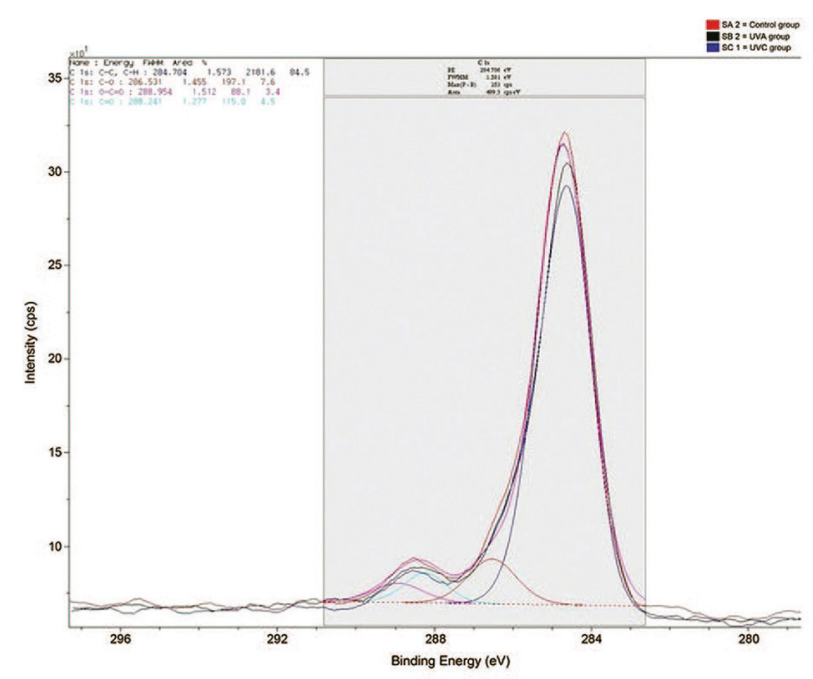

Fig. 3 X-ray photoelectron spectroscopy high-resolution spectra of the titanium surfaces of all the three groups showing $\mathrm{C} 1$ profile.

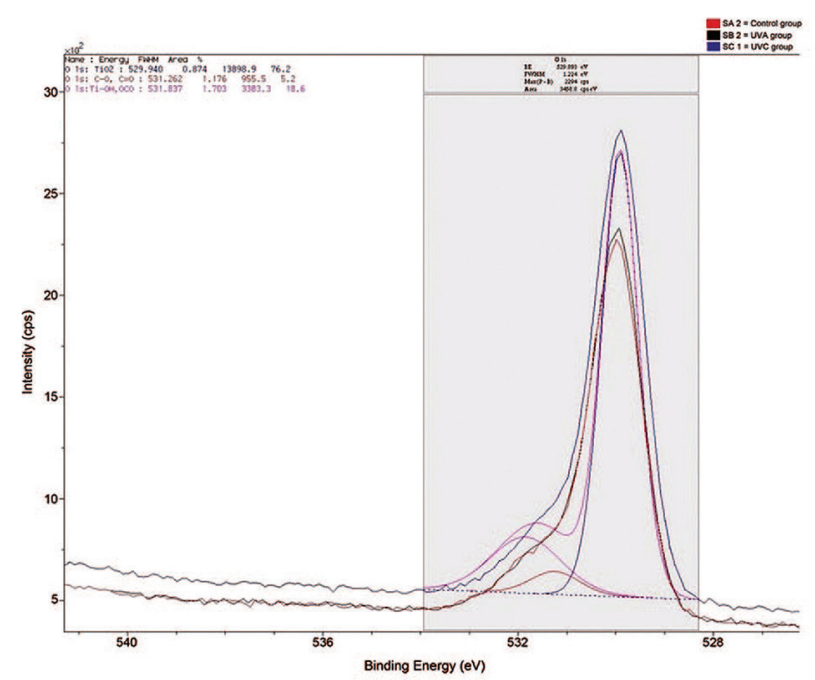

Fig. 4 X-ray photoelectron spectroscopy high-resolution spectra of the titanium surfaces of all the three groups showing 01 profile.

groups. ${ }^{11}$ A previous study showed that decreasing the percentage of carbon to $<20 \%$ in acid-etched implants caused maximum cellular proliferation and hydrophilicity on the surface. ${ }^{10}$ In this study, SLA implants were photofunctionalized for just $10 \mathrm{~min}$ with UVA ( $382 \mathrm{~nm}, 25 \mathrm{~mW} / \mathrm{cm}^{2}$ ) and UVC $\left(260 \mathrm{~nm}, 15 \mathrm{~mW} / \mathrm{cm}^{2}\right.$ ) irradiations. The important positive factor is that after irradiating the implants for a short period and at higher intensity, the carbon percentage decreased to approximately $25 \%$ in both experimental groups. Thus, a slight increase in the exposure time may decrease the carbon content to $<20 \%$ and further reduce $\mathrm{C} / \mathrm{Ti}$ ratios, which are much more feasible for dental chair-side procedures compared with treating the implants for $48 \mathrm{~h}$ as performed previously. ${ }^{11,12}$

Although both UVA and UVC irradiations can remove surface hydrocarbons, UVC is considered superior because it can improve hydrophilicity, protein absorption, and cell function. ${ }^{10,13}$ Remarkably, the effect on hydrocarbon levels on microarc oxidized (MAO) titanium disks after irradiating

\section{UV radiation}

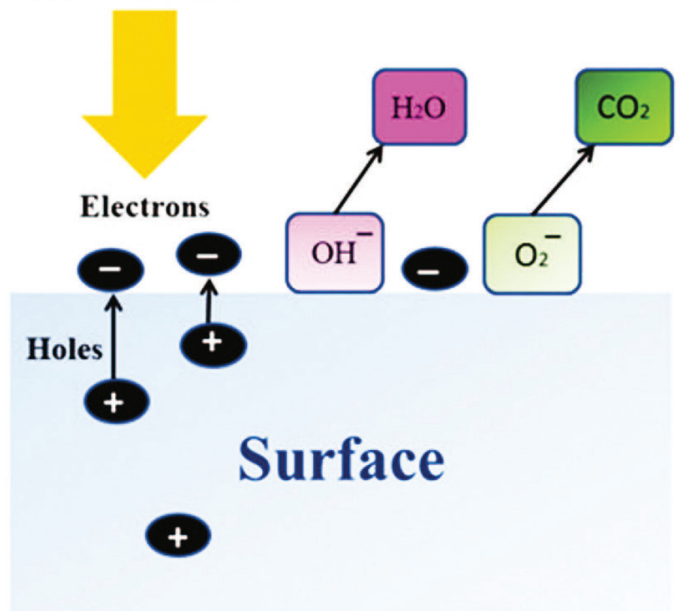

Fig. 5 The brief illustration of photocatalytic activity on surface.

$15 \mathrm{~W}$ UVA and UVC lamps for $24 \mathrm{~h}^{13}$ was almost the same as that observed in this study; alternatively, carbon atomic percentages decreased to a similar level in both UV-treated groups even after $10 \mathrm{~min}$ of irradiation.

Meanwhile, Ti-OH peaks in the 01s spectra showed an increasing trend in both irradiated groups ( - Fig. 4). Although Ti-OH levels were almost similar in both UV-treated groups, the peak oxygen intensity at $529.89 \mathrm{eV}$ increased for UVCtreated implants compared with that for UVA-treated implants. This difference in oxygen peaks proves that UVA and UVC irradiations employ different mechanisms for the removal of hydrocarbons. UVA irradiation removes surface hydrocarbons through photocatalysis, whereas UVC irradiation directly decomposes hydrocarbons through photolysis, generating more Ti-OH. ${ }^{14,15}$ During photocatalysis, UVA irradiation causes excitation of electrons, resulting in the release of various $\mathrm{OH}$ radicals and anionic oxygen species or superoxide radical anions. These reactive species further induce the photocatalytic decomposition of organic hydrocarbon molecules to the end products water and $\mathrm{CO}_{2}$ ( - Fig. 5). ${ }^{5}$ In photolysis, UVC irradiation converts $\mathrm{Ti}^{4+}$ ions to $\mathrm{Ti}^{3+}$ ions generating oxygen vacancies that react with absorbed water and form Ti-OH on the titanium surface. ${ }^{13}$ In this study, this phenomenon can be linked to a higher oxygen peak and a slightly more intense Ti2p peak in the UVC-treated group, probably due to photolysis. Lower titanium and oxygen peaks of group B showed inefficient oxygen, titanium, and $\mathrm{OH}$ radical production, concluding that UVA light causes photocatalytic hydrocarbon removal due to lower light intensity.

These findings from SLA-coated implants are similar to those of a prior study conducted using MAO-coated implants, ${ }_{16}^{16}$ proving that UVC photofunctionalization potentially induces greater surface energy and hydrophilicity on different implant surfaces. However, since the main focus was to evaluate the changes in carbon, titanium, and oxygen contents on titanium surfaces, further studies must be done to evaluate the cellular attachment changes after UVA and UVC treatment on SLA-coated implants. Using this standardized technique of photofunctionalization, the 
state of hydrocarbons and biological response of various implants with different surface coatings can be analyzed and compared.

\section{Conclusion}

In this study, SLA-etched surface titanium implants were exposed for 10 min using a standardized UVA and UVC irradiation through the UVACUBE 100. Although the decrease in surface hydrocarbons was the same in both of the UV-treated groups, the peak intensity of oxygen was increased in the UVC-treated group. Thus, we conclude that, compared with UVA irradiation, UVC irradiation has the potential to induce more hydrophilicity on SLA-coated implants.

\section{Financial Support and Sponsorship}

None.

\section{Conflicts of Interest}

None.

\section{Acknowledgment}

We thank Science and Engineering Research Centre, Universiti Sains Malaysia for providing technical support on XPS.

\section{References}

1 Kamo M, Kyomoto M, Miyaji F. Time course of surface characteristics of alkali- and heat-treated titanium dental implants during vacuum storage. J Biomed Mater Res B Appl Biomater 2017;105(6):1453-1460

2 Morra M, Cassinelli C, Bruzzone G, et al. Surface chemistry effects of topographic modification of titanium dental implant surfaces: 1. Surface analysis. Int J Oral Maxillofac Implants 2003;18(1):40-45

3 Yoshihara C, Ueno T, Chen P, Tsutsumi Y, Hanawa T, Wakabayashi $\mathrm{N}$. Inverse response of osteoblasts and fibroblasts to growth on carbon-deposited titanium surfaces. J Biomed Mater Res B Appl Biomater 2018;106(5):1869-1877

4 Hayashi R, Ueno T, Migita S, et al. Hydrocarbon deposition attenuates osteoblast activity on titanium. J Dent Res 2014;93(7):698-703
5 Rupp F, Liang L, Geis-Gerstorfer J, Scheideler L, Hüttig F. Surface characteristics of dental implants: a review. Dent Mater 2018;34(1):40-57

6 Henningsen A, Smeets R, Heuberger R, et al. Changes in surface characteristics of titanium and zirconia after surface treatment with ultraviolet light or non-thermal plasma. Eur J Oral Sci 2018;126(2):126-134

7 Att W, Ogawa T. Biological aging of implant surfaces and their restoration with ultraviolet light treatment: a novel understanding of osseointegration. Int J Oral Maxillofac Implants 2012;27(4):753-761

8 Li S, Ni J, Liu X, et al. Surface characteristics and biocompatibility of sandblasted and acid-etched titanium surface modified by ultraviolet irradiation: an in vitro study. J Biomed Mater Res B Appl Biomater 2012;100(6):1587-1598

9 Sackey SS, Vowotor MK, Owusu A, et al. Spectroscopic study of UV transparency of some materials. Environ Pollut 2015;4(4):1

10 Uchiyama H, Yamada M, Ishizaki K, Sakurai K. Specific ultraviolet-C irradiation energy for functionalization of titanium surface to increase osteoblastic cellular attachment. J Biomater Appl 2014;28(9):1419-1429

11 Aita H, Hori N, Takeuchi M, et al. The effect of ultraviolet functionalization of titanium on integration with bone. Biomaterials 2009;30(6):1015-1025

12 Yamazaki M, Yamada M, Ishizaki K, Sakurai K. Ultraviolet-C irradiation to titanium implants increases peri-implant bone formation without impeding mineralization in a rabbit femur model. Acta Odontol Scand 2015;73(4):302-311

13 Gao Y, Liu Y, Zhou L, et al. The effects of different wavelength UV photofunctionalization on micro-arc oxidized titanium. PLoS One 2013;8(7):e68086

14 Aita $\mathrm{H}$, Att $\mathrm{W}$, Ueno $\mathrm{T}$, et al. Ultraviolet light-mediated photofunctionalization of titanium to promote human mesenchymal stem cell migration, attachment, proliferation and differentiation. Acta Biomater 2009;5(8):3247-3257

15 Miyauchi T, Yamada M, Yamamoto A, et al. The enhanced characteristics of osteoblast adhesion to photofunctionalized nanoscale TiO2 layers on biomaterials surfaces. Biomaterials 2010;31(14):3827-3839

16 Son HS, Ko G, Zoh KD. Kinetics and mechanism of photolysis and TiO2 photocatalysis of triclosan. J Hazard Mater 2009; 166(2-3):954-960 